\title{
Determinação de Índices de Zona no Sistema Hexagonal
}

\author{
WILLIAM G. R. DE CAMARGO \\ (Departamento de Mineralogia e Petrografia, Universidade de S. Paulo)
}

\begin{abstract}
A general graphical solution of zone index determination in the hexagonal system is herein described. Zone axis may be treated as vectors in such a manner that they could be solved into several components, according to the crystallographic axis $\mathrm{X}, \mathrm{Y}, \mathrm{W}, \mathrm{Z}$. These components ard proportional to the searched zone indices.

The vector decomposition may be performed by drawing normals to the crystallographic axis from any given point $\mathrm{P}$ of the vector. In such way, the computed zone indices will satisfy the condition $u+v+\omega=0$.
\end{abstract}

Em trabalho anterior (Mineração e Metalurgia n. 85, v. XV, 1950) foi deduzida a relação $\mathrm{h}+\mathrm{k}+\mathrm{i}=0$, para os índices de face de um cristal hexagonal, e foi demonstrado como a relação $u+v+\omega=0$, poderia ser imposta e estabelecida por convenção, pois que para os índices de zona haveria um número indeterminado de soluções possíveis.

No mesmo trabalho, a solução gráfica da determinação dos índices de zona ou de aresta foi tratada, porém sòmente aplicável a uma aresta $z^{\prime}$, que estivesse contida no plano equatorial, isto é, no plano dos três eixos cristalográficos horizontais $\mathrm{X}, \mathrm{Y}, \mathrm{W}$.

O presente artigo focaliza o problema gráfico de maneira mais geral. Considerando-se um vetor genérico $z$, que representa a direção de uma aresta no cristal (fig. 1), a sua decomposição gráfica pode ser efetuada de acôrdo com as indicações que se seguem. Traça-se um plano perpendicular ao vetor $z$, a qualquer distância da origem (pois 
não interessa o módulo do vetor, mas exclusivamente a sua direção) e faz-se o rebatimento do plano $Z-z-z$ ' para o papel (figs. 1 e 2 ).

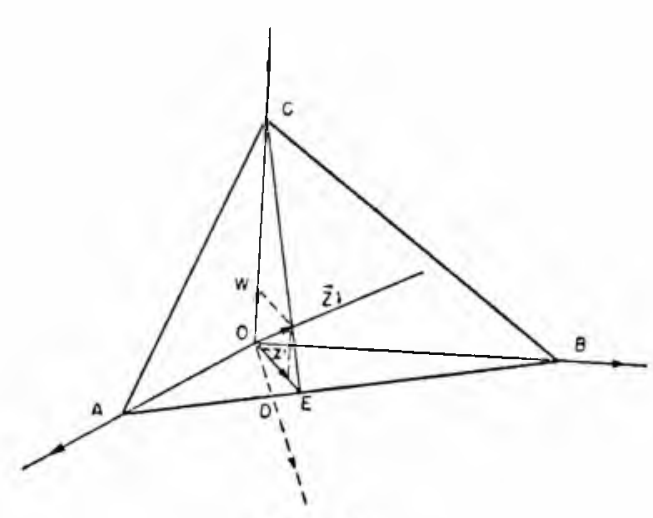

Fig. 1

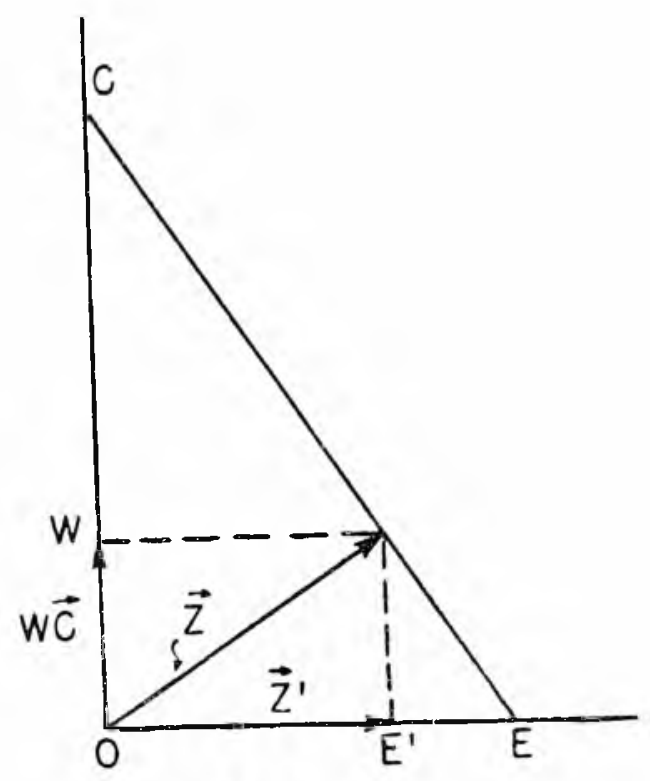

Fig. 2

O vetor $z$ deve ser decomposto em duas direções, pela regra do paralelogramo, obtendo-se uma componente OW segundo o eixo cristalográfico $Z$, e outra $z$ ' contida no plano horizontal. A componente $z$ ' possui direção variável, que depende de $z$ (fig. 1 ).

Para que u, v, $\omega$, índices de aresta ou zona, estejam em relação tal que quando recompostos conduzam novamente ao vetor $z$ primitivo, deve ser traçada uma perpendicular ao vetor $z$, passando pelo ponto E. Isto será feito para decompor $z^{\prime}$, de maneira a satisfazer a relação $u+v+\omega=0$ (fig. 3 ).

A perpendicular a $z^{\prime}$ no ponto $\mathrm{E}$, determina respectivamente sôbre cs eixos cristalográficos $\mathrm{X}, \mathrm{Y}, \mathrm{W}$, os segmentos $\mathrm{OA}, \mathrm{OB}$ e $\mathrm{OD}$, cujos inversos fornecem os índices $\mathrm{u}, \mathrm{v}, \omega$, como foi demonstrado em trabalho anterior. Êste processo gráfico, embora fàcilmente exequível, exige ainda pequeno trabalho analítico no cálculo dos inversos de $\mathrm{OA}, \mathrm{OB}$ e 
OD. Entretanto, um segundo processo inteiramente gráfico, pode ser demcnstrado para o cálculo dos inversos.

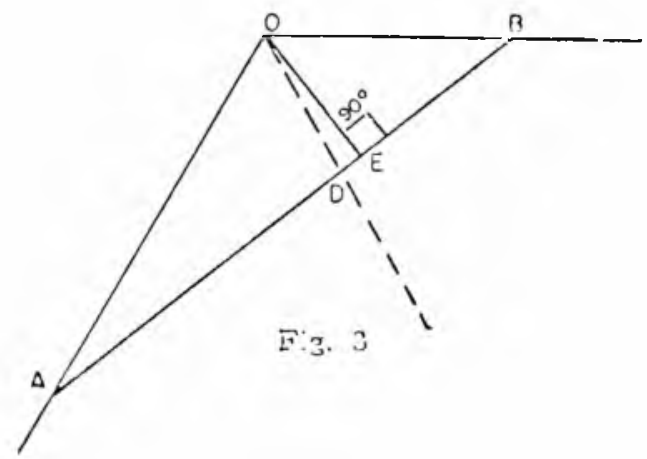

Dada uma determinada aresta definida pela direção do vetor $z$, êste pode ser decomposto em quatro componentes, segundo os eixos cristalográficos, traçando-se perpendiculares a $\mathrm{X}, \mathrm{Y}, \mathrm{W}, \mathrm{Z}$, a partir de um ponto $P$ qualqquer do vetor $z$ (fig. 4).

Os segmentos OU, OV, O, , OW, obtidos sôbre os eixos, são proporcionais a $\mathrm{u}, \mathrm{v}, \omega, \mathrm{w}$, e dão diretamente os índices de zona procurados, de forma a satisfazer $\mathrm{u}+\mathrm{v}+\boldsymbol{(})=0$. O segmento $\mathrm{OW}$, entretanto, deve ser dividido pela constante cristalográfica c, relativa ao eixo $Z$.

Os ângulos diretores da aresta, $\mathrm{Px}, \mathrm{Py}, \mathrm{Pw}_{\mathrm{w}}$ e $\mathbf{P z}$ devem ser calculados por processo analítico e constituem dados experimentais extraídos de medidas goniométricas de um determinado cristal.

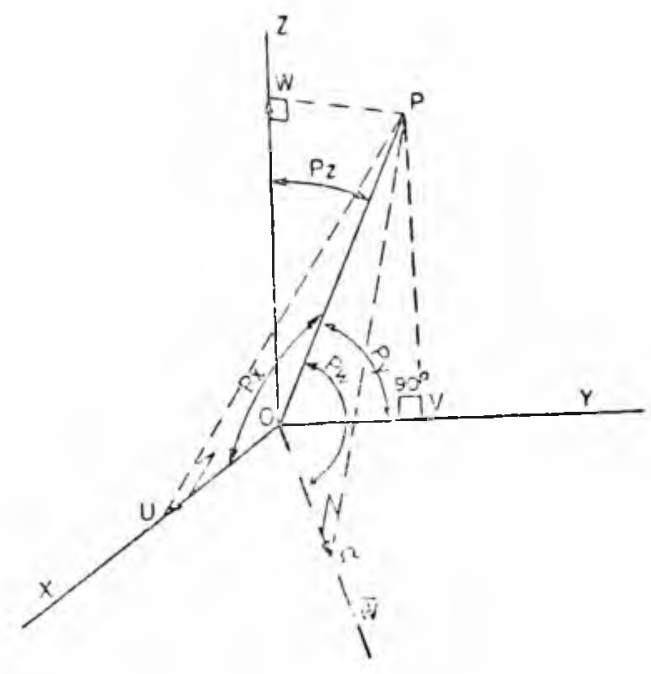

Fig. 7

A prova matemática do processo gráfico descrito, tratada de maneira mals geral, constitui ainda processo analítico para o cômputo de $\mathrm{u}, \mathrm{v}, \omega, \mathrm{w}$, na relação $\mathrm{u}+\mathrm{v}+\omega=0$. Se ficar provado, que para arestas e faces mùtuamente perpendiculares, é válida a seguinte relação, 
$\mathrm{h}: \mathrm{k}: \mathrm{i}=\mathrm{u}: \mathrm{v}:(\omega)$ a relação $\mathrm{u}+\mathrm{v}+\omega=0$ está automàticamente de. monstrada, pois que, como já foi demonstrado, $h+k+i=0$.

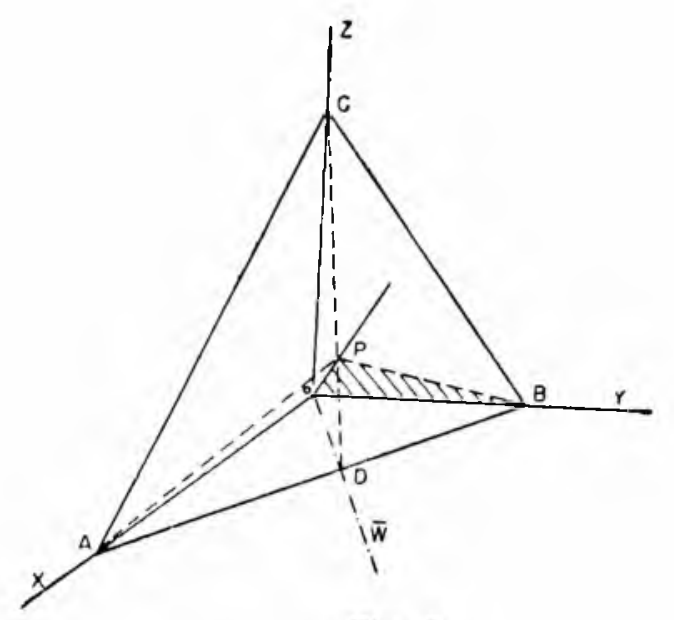

Fig. 5

Seja uma face (fig. 5) $A B C D$ e uma aresta perpendicular OP. Sejam ainda OA, OB, OC, OD, ou abreviadamente a', b', c', d', os parâmetros da face considerada.

No triângulo retângulo OPA:

$$
\begin{aligned}
& \mathrm{OP} / \mathrm{OA}=\cos \mathrm{Px} \\
& \mathrm{OP}=\mathrm{OA} \cos \mathrm{Px}=\mathrm{a}^{\prime} \cos \mathrm{Px}
\end{aligned}
$$

Por analogia:

$$
\begin{aligned}
& \mathrm{OP}=\mathrm{b}^{\prime} \cos \mathrm{Py} \\
& \mathrm{OP}=\mathrm{d}^{\prime} \cos \mathrm{Pw} \\
& \mathrm{OP}=\mathrm{c}^{\prime} \cos \mathrm{Pz}
\end{aligned}
$$

Portanto:

$$
\mathrm{a}^{\prime}: \mathrm{b}^{\prime}: \mathrm{d}^{\prime}: \mathrm{c}^{\prime}=1 / \cos \mathrm{Px}: 1 / \cos \mathrm{Py}: 1 / \cos \mathrm{Pw}: 1 / \cos \mathrm{Pz}
$$

Pela relação de Hauy aplicada ao sistema hexagonal:

$$
\begin{aligned}
& \mathrm{h}: \mathrm{k}: \mathrm{i}: 1=1 / \mathrm{a}^{\prime}: 1 / \mathrm{b}^{\prime}: 1 / \mathrm{d}^{\prime}: \mathrm{c} / \mathrm{c}^{\prime} \\
& \mathrm{h}: \mathrm{k}: \mathrm{i}: 1=\cos \mathrm{Px}: \cos \mathrm{Py}: \cos \mathrm{P} \mathrm{w}: \mathrm{c} \operatorname{cosPz}
\end{aligned}
$$

Nos triângulos retângulos OPU, OPV, OP $\Omega$, OPW, formados pela decomposição do vetor $z$ (fig. 4):

$$
\begin{aligned}
& \mathrm{OU} / \mathrm{OP}=\cos \mathrm{Px} \\
& \mathrm{u}=\mathrm{OU}=\mathrm{OP} \cos \mathrm{Px}
\end{aligned}
$$

Por analogia:

$$
\begin{aligned}
& \mathrm{v}=\mathrm{OP} \cos \mathrm{Py} \\
& \mathrm{\omega}=\mathrm{OP} \cos \mathrm{Pw} \\
& \mathrm{w}=\mathrm{OP} \cos \mathrm{Pz}
\end{aligned}
$$


Relacionando $\mathrm{u}: \mathrm{v}: \omega: \mathrm{w}$, teremos:

$$
\mathrm{u}: \mathrm{v}: \omega: \mathrm{w}=\cos \mathrm{P} \mathrm{x}: \cos \mathrm{Py}: \cos \mathrm{Pw}: \cos \mathrm{Pz} / \mathrm{c}
$$

Comparando as expressões (1) e (2), veremos que para os primeiros três índices vale a seguinte equação:

$\mathrm{u}: \mathrm{v}: \omega=\mathrm{h}: \mathrm{k}: \mathrm{i}$

Portanto se existir uma relação $h+k+i=0$, como já foi comprovado, haverá anàlogamente $u+v+\omega=0$, desde que a decomposição dos vetores seja efetuada conforme processo descrito.

\section{BIBLIOGRAFIA}

Camargo, W. G. R. (1950), Os índices de face e de zona no sistema hexagonal. Mineração e Metalurgia, vol. XV, n. 85.

TAVORA, E. (1946), Solução vetorial para uma conversão de símbolos. Mineração e Metalurgia, v. 11, n. 61, pg. 31.

Niggli, P. (1924), Lehrbuch der Mineralogie, pp. 107-120,Bd. I, Berlin. Wolff, C. W., (1944), Hexagonal zone symbols and transformation formulae - Am. Min., v. 29, n. 1-2, pp. 49-55.

HEy, M. H. (1930), On face and zone symbols referred to the hexagonal axis. Min. Mag., vol. 22, pg. 283.

Werber, L. (1922), Zt. f. Kr., vol. 57, pg. 200.

Camargo, W. G. R. (1952), Miller indices in the hexagonal system (no prelo). 